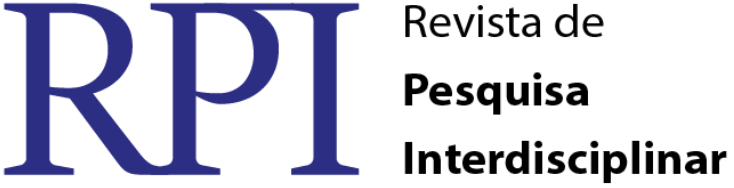

\section{ABORDAGEM INTERDISCIPLINAR DE EDUCAÇ̃̃O EM SAÚDE: A PRÁTICA DA AUTOMEDICAÇÃO ENTRE UNIVERSITÁRIOS}

\author{
Patrícia da Silva Sousa - UFCA - IFE ${ }^{1}$ \\ Francisca Cinthia de Oliveira - UFCA - IFE ${ }^{2}$ \\ Laura Hévila Inocêncio Leite - UFCA - IFE ${ }^{3}$
}

\begin{abstract}
RESUMO
O presente trabalho tem como objetivo mostrar os riscos da automedicação, ou seja, o uso de medicamentos por conta própria ou por indicação de outras pessoas não habilitadas para o tratamento de doenças cujos sintomas são de fácil percepção pelo usuário. Neste sentindo foi feito uma verificação sobre o uso de medicamentos entre os universitários da Universidade Federal do Cariri (UFCA) do curso de Licenciatura Interdisciplinar em Ciências Naturais e Matemática, a fim de esclarecer os possíveis motivos dos mesmos preferirem se automedicar, ao invés de procurar um médico. Apontando alguns medicamentos que fazem uso frequentemente sem a orientação de um profissional. Foram realizadas entrevistas aleatórias com 14 estudantes, de ambos os sexos e entre uma faixa etária de 17 a 60. Os resultados deste levantamento revela a realidade de muitas pessoas, que fazem uso indiscriminado de medicamentos por não terem tempo de ficar na fila de um hospital com sintomas que podem ser facilmente tratados em casa, ou por não terem plano de saúde e condições para pagar um. São fatores que influência, além da disponibilidade de produtos no mercado que gera maior familiaridade do usuário leigo ao uso de medicamentos. Avaliando os resultados da recolha dos 14 entrevistados entre eles nove mulheres e cinco homens, mulheres têm maiores índices do uso de medicamentos, fazendo uso frequentemente de analgésicos para dor, antigripal para gripe, e antiinflamatório para inflamação, dor e febre sendo uns dos mais procurados por ambos os entrevistados. Com isso espera-se que trabalhos futuros apresente para os entrevistados uma nova visão sobre como usar e adquirir medicamentos de forma a não colocarem em risco a sua saúde, pelo uso irracional de fármacos.
\end{abstract}

Palavras-Chaves: Automedicação, Uso Irracional, Fármacos.

\section{INTERDISCIPLINARY APPROACH FOR HEALTH EDUCATION: THE PRACTICE OF AUTOMEDICATION BETWEEN UNIVERSITARIANS}

\begin{abstract}
This work aims to show the risks of self-medication, or medication use on their own or indication of other persons not authorized for the treatment of diseases whose symptoms are easily understood by the user. This feeling was made a check on the use of drugs among college students of the Federal

\footnotetext{
1 Dicente do curso de Licenciatura em Ciências Naturais e Matemática UFCA - IFE campus Brejo Santo,*patys.silvasousa@gmail.com

2 Dicente do curso de Licenciatura em Ciências Naturais e Matemática UFCA - IFE campus Brejo Santo,*patys.silvasousa@gmail.com

3 Docente do curso de Licenciatura em Ciências Naturais e Matemática UFCA - IFE campus Brejo Santo, *laura.leite@ufca.edu.br
} 
University of Cariri (UFCA) course Interdisciplinary Degree in Natural Sciences and Mathematics in order to clarify the possible reasons of the same prefer to self-medicate, instead of seeking a doctor. Pointing some medications that make use often without the guidance of a professional. Random interviews were conducted with 14 students of both sexes and between age 17 to 60 . The results of this survey reveals the reality of many people who make indiscriminate use of drugs not have time to stand in line at a hospital with symptoms that can be easily treated at home, or do not have health insurance and conditions for pay. Factors that influence, and the availability of products on the market that generates greater familiarity of the lay user to drug use. Evaluating the results of the collection of the 14 respondents including nine women and five men, women have higher rates of drug use, using often analgesics for pain, flu to influenza, and anti-inflammatory for inflammation, pain and fever being one of most sought after by both respondents. It is expected that future work present to respondents a new vision on how to use and purchase medicines so as not to jeopardize their health, the irrational use of drugs

Keywords: Self-Medication, Irrational, Use Drugs.

\section{INTRODUÇÃO}

Os medicamentos ocupam papel importante na sociedade, pois é responsável por salvar vidas, são de grande importância no sistema de saúde e quando usado de maneira correta pode tornar-se um recurso viável para o tratamento de doenças crônicas. Porém condutas que resultam no uso irracional de medicamentos podem acarretar consequências graves á saúde da população, como: reações adversas, dependências ao medicamento, e intoxicação por fazer uso incorreto de medicação.

De acordo com a Organização Mundial de Saúde (2008), o uso irracional de medicamentos envolve vários hábitos que incluem: a utilização simultânea de muitos medicamentos sem critérios técnicos, o uso inapropriado de classes farmacológicas e prescrições médicas inadequadas. Diante da necessidade do uso criterioso de medicamentos, o farmacêutico torna- se responsável por contribuir com o uso racional.

O amplo uso de medicamentos, sempre vem desconhecido dos seus malefícios. O acumulo de fármacos nas residências, é um grande fator de riscos, pois favorece confusões na hora de fazer a automedicação.

O uso indiscriminado de medicação sem prescrição médica é uma questão que envolve educação em saúde.

RPI Revista de Pesquisa Interdisciplinar, Cajazeiras, v. 1, Ed. Especial, 106 - 113, set/dez. de 2016. 
A interdisciplinaridade, nesse contexto, caracteriza-se como integração entre diferentes áreas, sendo que a abordagem de problemas de forma criativa demanda mudanças individuais, institucionais e ações Inter setoriais. Portanto, as práticas de saúde e as ações educativas tornam-se essenciais uma vez que agrupam estratégias para o estabelecimento de uma relação de reciprocidade, de mutualidade, de interação que possibilita o diálogo, o aprendizado e a participação de todos na realização dessas atividades (GALINDO; GOLDENBERG, 2008).

Tendo em vista, os fatores que levam o individuo a aderir essa prática, que embora simples e muitas vezes espontânea no cotidiano e as consequências decorrentes deste fato, o trabalho volta-se para os universitários da UFCA, onde buscou avaliar a percepção dos discentes através de um questionário direcionado ao público de diferentes idades, abordando questões visíveis à realidade de boa parte das pessoas, tais como: a frequência do uso de medicamentos, os motivos que os levam a ingerir sem curiosidade de se ter informações sobre essa ação, pois simples analgésicos podem trazer consequências graves para a saúde.

\section{Desenvolvimento}

A pesquisa foi feita com 14 universitários, do curso de Licenciatura Interdisciplinar em Ciências Naturais e Matemática. Estes eram convidados a responderem um questionário, sobre uso de medicamentos sem prescrição médica. Nenhum aluno era identificado, e a escolha foi feita aleatoriamente, priorizando estudantes do curso, nas faixas etárias de 17 a 25 anos, ambos os sexos. Após, devolução dos questionários, um estudo de outros artigos sobre a temática foi feito, a fim de cruzar os dados, e dar respaldo para a elaboração do trabalho.

Os medicamentos utilizados em geral possuem mais de uma única ação específica. Quando administrados a um paciente são absorvidos e distribuídos, ocorrendo normalmente a resposta esperada. (CLAYTON; STOK, 2006).

Esses medicamentos, quando administrados de forma indevida podem ocasionar diversos transtornos, as reações adversas ou efeitos colaterais.

A partir de várias descobertas, seguiu-se a introdução de novas técnicas e produtos, dando início à terapêutica de importante compostos de ação

RPI Revista de Pesquisa Interdisciplinar, Cajazeiras, v. 1, Ed. Especial, 106 - 113, set/dez. de 2016. 
analgésica, antipirética e anti-inflamatória, que continuam em franco desenvolvimento até os dias atuais. (SILVA, 2006).

Em consulta feita com os universitários de Brejo Santo, é de costume prévio os mesmo administrar anti-inflamatórios e analgésicos com mais continuidade e sem prescrição médica.

Com base nos dados da sondagem feita com os discentes, percebe- se que a ação de se automedicar por conta própria é um fator comum entre a maioria, nesse sentido o questionário continha oito perguntas objetivas contendo os seguintes conteúdos: Toma medicamentos por conta própria; por que toma por conta própria; se tem plano de saúde; se faz consulta médica nos últimos 06 meses; quais medicamentos que você usa; se tem conhecimento dos possíveis efeitos adversos; como tem conhecimento dos efeitos colaterais; costuma ler a bula do medicamento.

Em se tratando de educação em saúde, a conscientização é um dos aspectos em que a mudança de hábitos dos indivíduos, poderá aumentar o bem estar e a qualidade de vida, pois o objetivo é a redução dos malefícios proporcionados pelas doenças. Neste contexto, os universitários assume a responsabilidade de serem disseminadores de conhecimentos como os que envolvem a questão de educação em saúde.

Por fim, é importante relatar que os fatores que interfere, na utilização de medicamentos pelos universitários despertam preocupação, pois, a utilização irracionais destes produtos gera prejuízos econômicos e sociais. Como pode ser observados nos gráficos abaixo, a necessidade de ações interdisciplinares deve ser vista para a promoção do uso racional de medicamentos.

\section{Conclusão}

A partir da problemática encontrada, no campus da UFCA, visou-se a discussão e o debate para possíveis intervenções no contexto, de modo que os discentes pudessem ter uma visão voltada á conscientização da utilidade de medicamentos, a importância de conhecer seus benefícios e os malefícios quando administrados de maneira incorreta, que é um dos objetivos do campus por se tratar de um Instituto de Formação de Educadores. Quando se fala em automedicação, pensa-se em pessoas sem informação dos riscos dessa prática. No entanto, o que se percebe por meio deste trabalho, é que os universitários, tem essa prática de forma corriqueira.

RPI Revista de Pesquisa Interdisciplinar, Cajazeiras, v. 1, Ed. Especial, 106 - 113, set/dez. de 2016. 
Sendo assim, intencionalmente o proposto busca intervir significativamente na percepção dos universitários com relação a esse âmbito que muitas vezes é desconsiderado frente à idealização de uma conduta inadequada á população que precisaria priorizar a ética e preceitos de uma sociedade consciente. Segue em anexo, portanto, os gráficos com os resultados deste trabalho:

\section{REFERÊNCIAS}

ABRAÃO, Lígia Maria; SIMAS, José M. Marques; MIGUEL, Tatiana Longo Borges. Incidência da automedicação e uso indiscriminado de medicamentos entre jovens universitários. P.2-3, 2009.

CLAYTON, B. D.; STOCK, Y. N.; Farmacologia na prática de enfermagem. 13. ed. Rio de Janeiro: Elsevier, 2006.

GALINDO, M. B.; GOLDENBERG, P. Interdisciplinaridade na graduação em enfermagem: um processo de construção. Revista Brasileira de enfermagem, v. 61, n. 1, p. 18-23, 2008.

SILVA, P. Farmacologia. 7. ed. Rio de Janeiro: Guanabara Koogan, 2006.

ANEXO

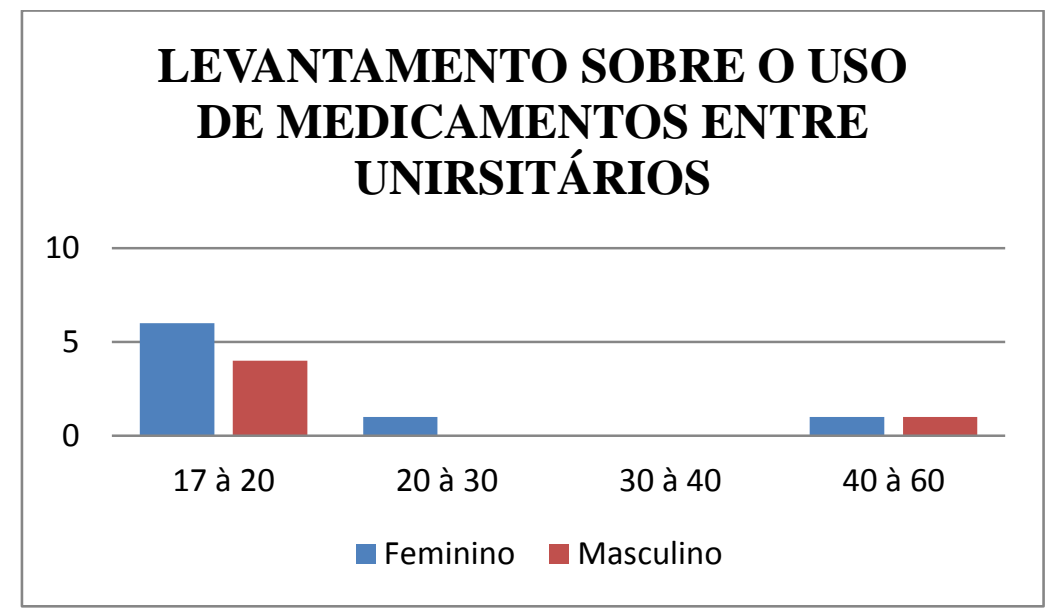

RPI Revista de Pesquisa Interdisciplinar, Cajazeiras, v. 1, Ed. Especial, 106 - 113, set/dez. de 2016. 


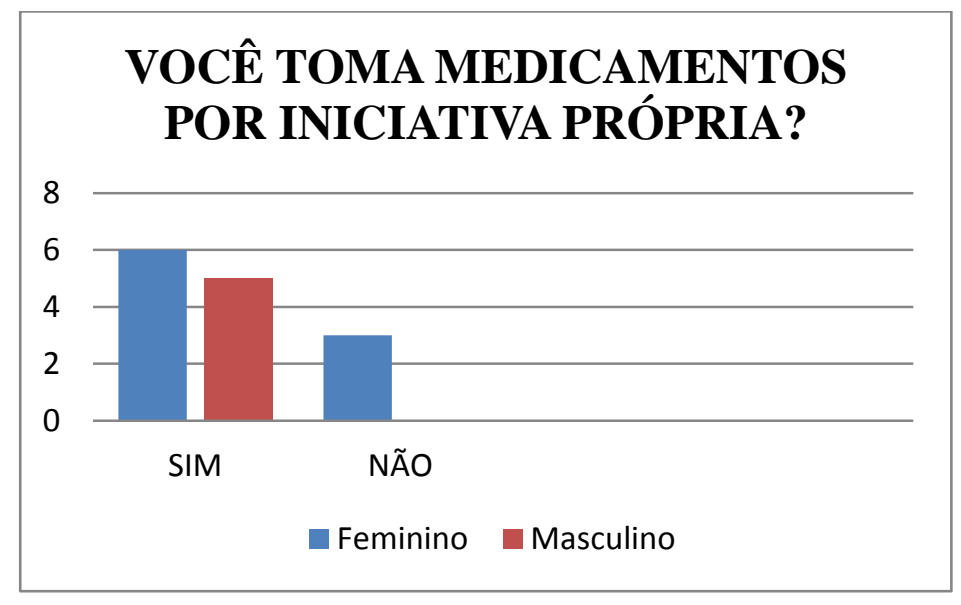

\section{Porque você toma medicamento por conta própria:}

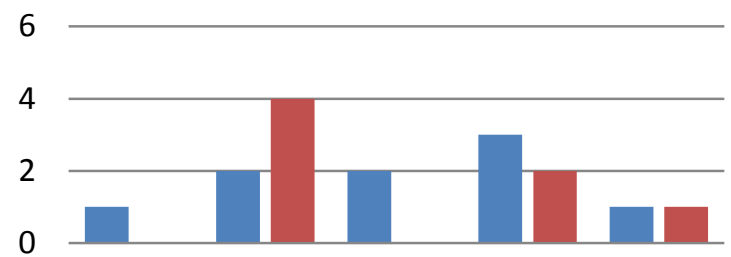

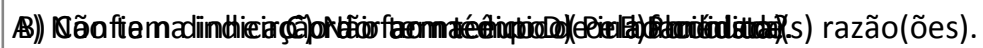

Feminino Masculino

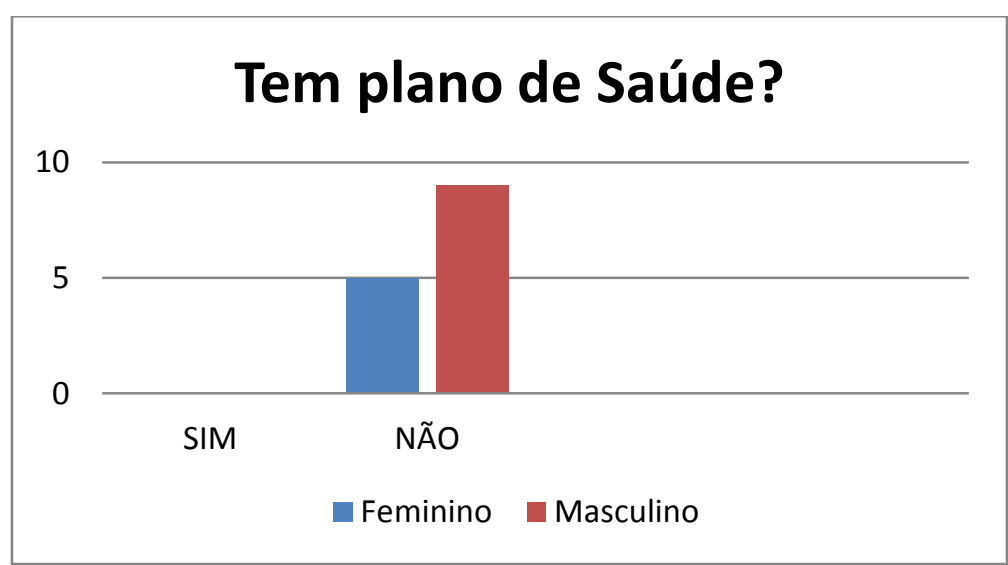

RPI Revista de Pesquisa Interdisciplinar, Cajazeiras, v. 1, Ed. Especial, 106 - 113, set/dez. de 2016. 

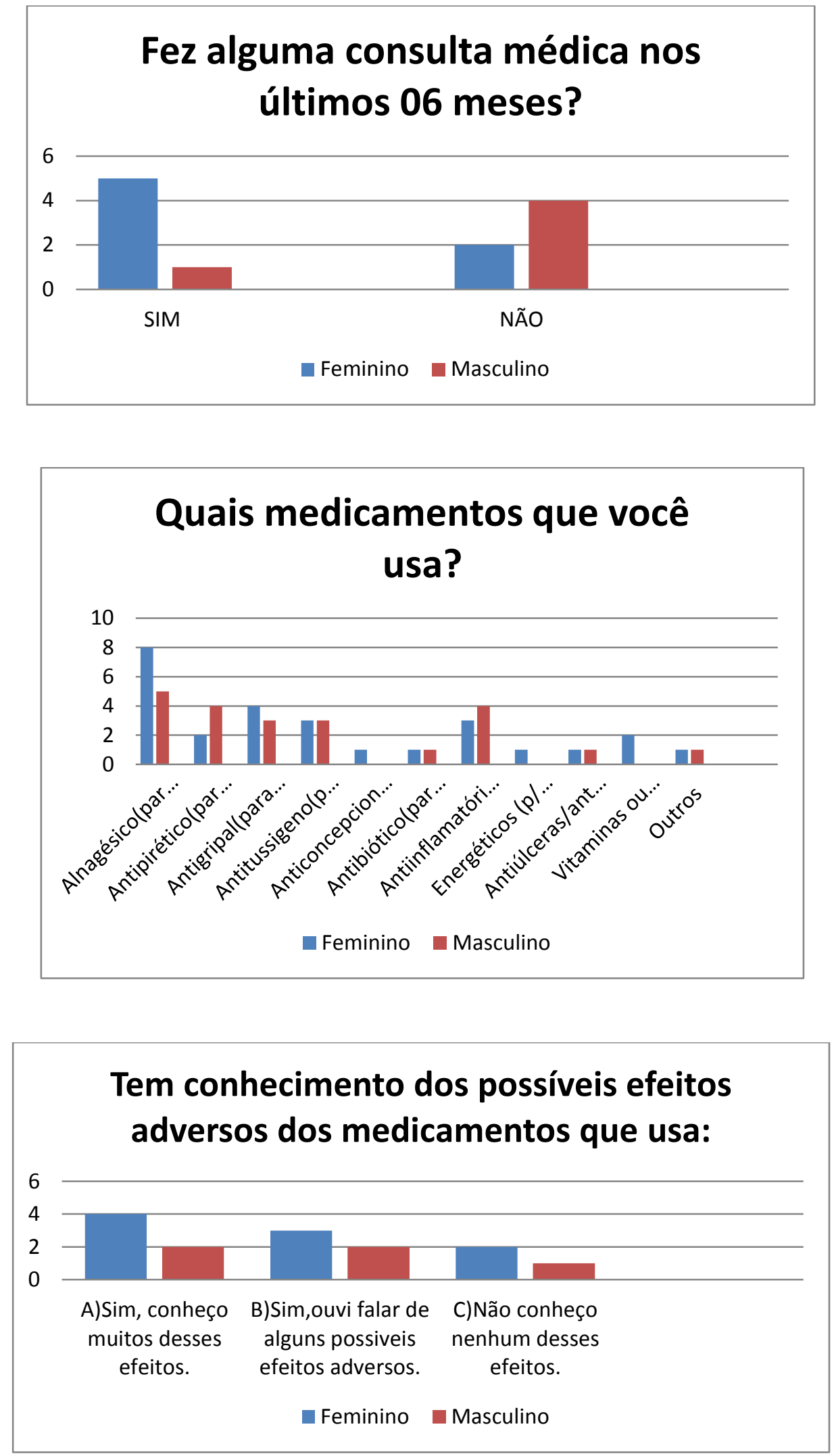

RPI Revista de Pesquisa Interdisciplinar, Cajazeiras, v. 1, Ed. Especial, 106 - 113, set/dez. de 2016. 


\section{Tem conhecimento dos possíveis efeitos adversos dos medicamentos} que usa:

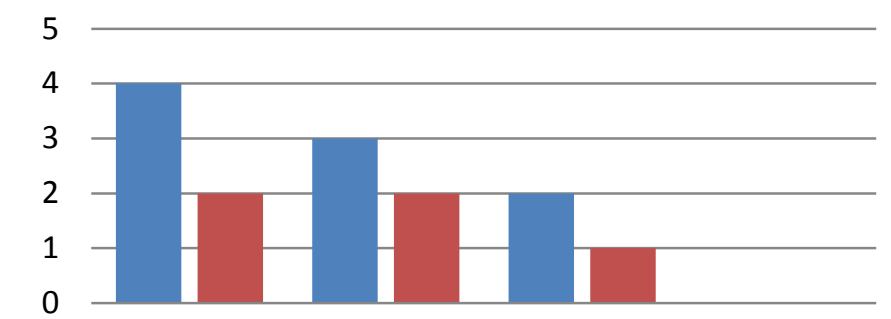

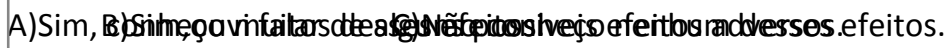

$$
\text { Feminino Masculino }
$$

\section{Se sua resposta foi (sim) na pergunta} anterior, como você tomou conhecimento desses efeitos:

6

4

2

0

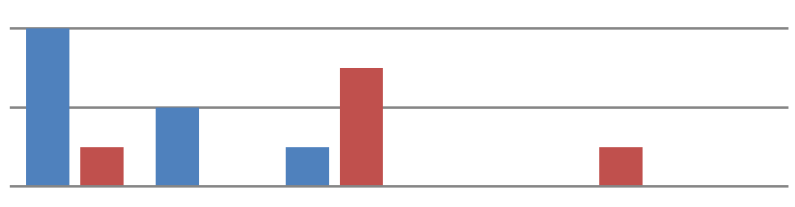

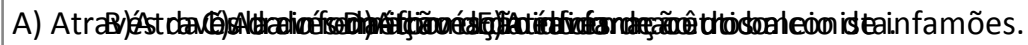

- Feminino Masculino

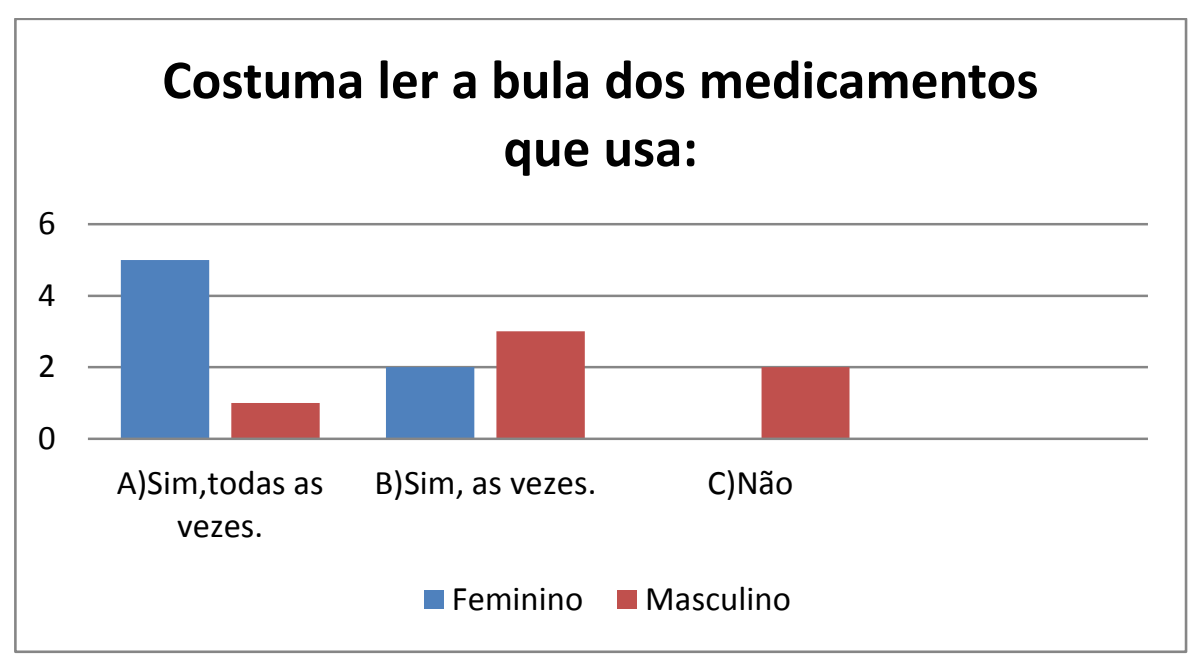

RPI Revista de Pesquisa Interdisciplinar, Cajazeiras, v. 1, Ed. Especial, 106 - 113, set/dez. de 2016. 